\title{
Canonical Ordering for Triangulations on the Cylinder, with Applications to Periodic Straight-Line Drawings
}

\author{
Luca Castelli Aleardi $^{1, \star}$, Olivier Devillers ${ }^{2}$, and Éric Fusy ${ }^{1, \star}$ \\ 1 LIX - École Polytechnique \\ 2 INRIA Sophia Antipolis - Méditerranée \\ \{amturing, fusy\}@lix.polytechnique.fr, olivier.devillers@inria.fr
}

\begin{abstract}
We extend the notion of canonical orderings to cylindric triangulations. This allows us to extend the incremental straight-line drawing algorithm of de Fraysseix, Pach and Pollack to this setting. Our algorithm yields in linear time a crossing-free straight-line drawing of a cylindric triangulation $G$ with $n$ vertices on a regular grid $\mathbb{Z} / w \mathbb{Z} \times[0 . . h]$, with $w \leq 2 n$ and $h \leq n(2 d+1)$, where $d$ is the (graph-) distance between the two boundaries. As a by-product, we can also obtain in linear time a crossing-free straight-line drawing of a toroidal triangulation with $n$ vertices on a periodic regular grid $\mathbb{Z} / w \mathbb{Z} \times \mathbb{Z} / h \mathbb{Z}$, with $w \leq 2 n$ and $h \leq 1+n(2 c+1)$, where $c$ is the length of a shortest non-contractible cycle. Since $c \leq \sqrt{2 n}$, the grid area is $O\left(n^{5 / 2}\right)$. Our algorithms apply to any triangulation (whether on the cylinder or on the torus) that have no loops nor multiple edges in the periodic representation.
\end{abstract}

\section{Introduction}

The problem of efficiently computing straight-line drawings of planar graphs has attracted a lot of attention over the last two decades. Two combinatorial concepts for planar triangulations turn out to be the basis of many classical straight-line drawing algorithms: the canonical ordering (a special ordering of the vertices obtained by a shelling procedure) and the closely related Schnyder wood (a partition of the inner edges of a triangulation into 3 spanning trees with specific incidence conditions). Algorithms based on canonical ordering [6]10] are typically incremental, adding vertices one by one while keeping the drawing planar. Algorithms based on Schnyder woods [14] are more global, the (barycentric) coordinates of each vertex have a clear combinatorial meaning (typically the number of faces in certain regions associated to the vertex). Algorithms of both types make it possible to draw in linear time a planar triangulation with $n$ vertices on a grid of size $O(n \times n)$. They can also both be extended to obtain (weakly) convex drawings of 3-connected maps on a grid of size $O(n \times n)$. The problem of obtaining planar drawings of higher genus graphs has been addressed less

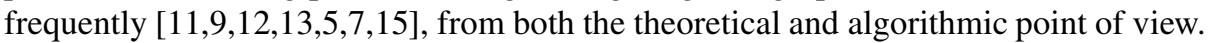
Recently some methods for the straight-line planar drawing of genus $g$ graphs with polynomial grid area (of size $O\left(n^{3}\right)$, in the worst case) have been described in [5]7] (to

\footnotetext{
* These authors are partially supported by the European project ExploreMaps -ERC StG 208471, and by the ANR grant EGOS 12 JS02 00201.
} 
apply these methods the graph needs to be unfolded planarly along a cut-graph). However, it does not yield (at least easily) periodic representations: for example, in the case of a torus, the boundary vertices (on the boundary of the rectangular polygon) might not be aligned, so that the drawing does not give rise to a periodic tiling.

Our main contribution is to generalize the notion of canonical ordering and the incremental straight-line drawing algorithm of de Fraysseix, Pach and Pollack [6] (shortly called FPP algorithm thereafter) to triangulations on the cylinder. For any triangulation $G$ of the cylinder, our algorithm yields in linear time a crossing-free straight-line drawing of $G$ on a regular grid (on the flat cylinder) of the form $\mathbb{Z} / w \mathbb{Z} \times[0 . . h]$, with $w \leq 2 n$ and $h \leq n(2 d+1)$, where $n$ is the number of vertices of $G$ and $d$ is the (graph-) distance between the two boundaries of $G$. By a reduction to the cylindric case (the reduction is done with the help of a so-called tambourine [2]), we also get a drawing algorithm on the torus. Precisely, for any toroidal triangulation $G$, we can obtain in linear time a crossing-free straight-line drawing of $G$ on a regular grid (on the flat torus) $\mathbb{Z} / w \mathbb{Z} \times \mathbb{Z} / h \mathbb{Z}$, with $w \leq 2 n$ and $h \leq 1+n(2 c-1)$, where $n$ is the number of vertices of $G$ and $c$ is the length of a shortest non-contractible cycle. Since $c \leq(2 n)^{1 / 2}$ as shown in [1], we have $h \leq(2 n)^{3 / 2}$, so that the grid area is $O\left(n^{5 / 2}\right)$.

For the toroidal case we mention that a notion of canonical ordering has been introduced in [4] (this actually works in any genus and yields an efficient encoding procedure) but we do not use it here. We also mention that, independently, an elegant periodic straight-line drawing algorithm for toroidal triangulations has been very recently described in [8], based on so-called toroidal Schnyder woods and face-counting operations; in their case the area of the periodic grid is $O\left(n^{4}\right)$.

\section{Preliminaries}

Graphs embedded on surfaces. A map of genus $g$ is a connected graph $G$ embedded on the compact orientable surface $S$ of genus $g$, such that all components of $S \backslash G$ are topological disks, which are called the faces of the map. The map is called planar for $g=0$ (embedding on the sphere) and toroidal for $g=1$ (embedding on the torus). The dual of a map $G$ is the map $G^{*}$ representing the adjacencies of the faces of $G$, i.e., there is a vertex $v_{f}$ of $G^{*}$ in each face $f$ of $G$, and each edge $e$ of $G$ gives rise to an edge $e^{*}=\left\{v_{f}, v_{f^{\prime}}\right\}$ in $G^{*}$, where $f$ and $f^{\prime}$ are the faces on each side of $e$. A cylindric map is a planar map with two marked faces $B_{1}$ and $B_{2}$ whose boundaries $C\left(B_{1}\right)$ and $C\left(B_{2}\right)$ are simple cycles (possibly $C\left(B_{1}\right)$ and $C\left(B_{2}\right)$ share vertices and edges). The faces $B_{1}$ and $B_{2}$ are called the boundary-faces. Boundary vertices and edges are those belonging to $C\left(B_{1}\right)$ (black circles in Fig. 1) or $C\left(B_{2}\right)$ (gray circles in Fig. 1); the other ones are called inner vertices (white circles in Fig.11) and edges.

Periodic drawings. Here we consider the problem of drawing a cylindric triangulation on the flat cylinder and drawing a toroidal triangulation on the flat torus. For $w>0$ and $h>0$, the flat cylinder of width $w$ and height $h$ is the rectangle $[0, w] \times[0, h]$ where the vertical sides are identified. A point on this cylinder is located by two coordinates $x \in \mathbb{R} / w \mathbb{Z}$ and $y \in[0, h]$. The flat torus of width $w$ and height $h$ is the rectangle $[0, w] \times[0, h]$ where both pairs of opposite sides are identified. A point on this torus is located by two coordinates $x \in \mathbb{R} / w \mathbb{Z}$ and $y \in \mathbb{R} / h \mathbb{Z}$. Assume from now on that $w$ and 

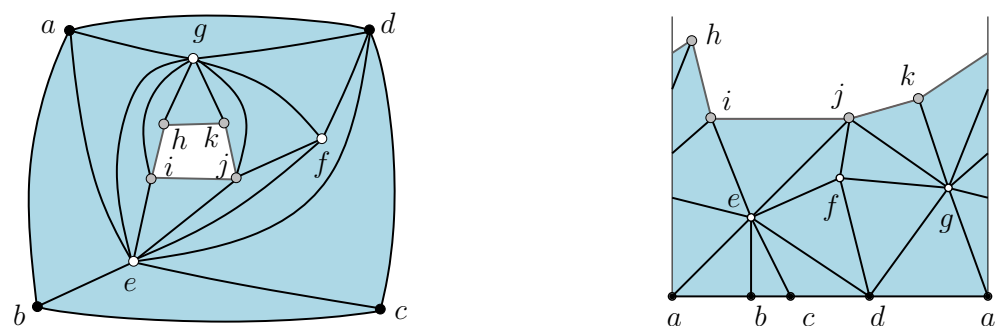

Fig. 1. A cylindric triangulation with boundary faces $B_{1}=\{a, b, c, d\}$ and $B_{2}=\{h, i, j, k\}$. Left: annular representation. Right: $x$-periodic representation.

$h$ are positive integers. For a cylindric triangulation $G$, a periodic straight-line drawing of $G$ of width $w$ and height $h$ is a crossing-free straight-line drawing (edges are drawn as segments, two edges can meet only at common end-points) of $G$ on the flat cylinder of width $w$ and height $h$, such that the vertex-coordinates are in $\mathbb{Z} / w \mathbb{Z} \times[0 . . h]$ (i.e., are integers). Similarly for a toroidal triangulation $G$, a periodic straight-line drawing of $G$ of width $w$ and height $h$ is a crossing-free straight-line drawing (edges are drawn as segments, two edges can meet only at common end-points) of $G$ on the flat torus of width $w$ and height $h$, such that the vertex-coordinates are in $\mathbb{Z} / w \mathbb{Z} \times \mathbb{Z} / h \mathbb{Z}$ (i.e., are integers).

\section{Periodic Drawings of Cylindric Triangulations}

A cylindric triangulation is a cylindric map with no loops nor multiple edges and such that all non-boundary faces are triangles (in Section 5 we will allow such triangulations to have 1-cycles or 2-cycles separating the two boundary-faces). We introduce at first a notion of canonical ordering for cylindric triangulations:

Definition 1. Let $G$ be a cylindric triangulation with boundary-faces $B_{1}$ and $B_{2}$, and such that the cycle $C\left(B_{1}\right)$ has no chords (i.e., there is no edge that is not on $C\left(B_{1}\right)$ and has both ends on $\left.C\left(B_{1}\right)\right)$. An ordering $\pi=\left\{v_{1}, v_{2}, \ldots, v_{n}\right\}$ of the vertices of $G \backslash C\left(B_{1}\right)$ is called a (cylindric) canonical ordering if it satisfies:

- For each $k \geq 0$ the map $G_{k}$ induced by $C\left(B_{1}\right)$ and by the vertices $\left\{v_{1}, \ldots, v_{k}\right\}$ is a cylindric triangulation.

- The other boundary-face $B^{\prime}$ of $G_{k}$ (the one different from $B_{1}$ ), whose contour is denoted $C_{k}$, contains $B_{2}$ (to clarify the meaning of “ $B^{\prime}$ contains $B_{2}$ ”, remember that a cylindric map is a special kind of map on the sphere; since $G_{k}$ is a submap of $G$, any face of $G_{k}$ is made of a set of faces of $G$ ).

- The vertex $v_{k}$ is on $C_{k}$, and its neighbours in $G_{k-1}$ are consecutive on $C_{k-1}$.

The notion of canonical ordering makes it possible to construct a cylindric triangulation $G$ incrementally, starting from $G_{0}=C\left(B_{1}\right)$ and adding one vertex (and incident edges) at each step. This is similar to canonical orderings for planar triangulations, as 
introduced by de Fraysseix, Pach and Pollack [6] (the main difference is that for a planar triangulation one starts with $G_{0}$ being an edge, called the base-edge, whereas here one starts with $G_{0}$ being a cycle, seen as a cylindric map without non-boundary faces).

Shelling Procedure. We now describe a shelling procedure to compute a canonical ordering of a cylindric triangulation $G$ with boundary-faces $B_{1}, B_{2}$. At each step the graph formed by the remaining vertices is a cylindric triangulation, one boundary face remains $B_{1}$ all the way, while the other boundary-face (initially $B_{2}$ ) has its contour, denoted by $C_{k}$, getting closer to $C\left(B_{1}\right)$. A vertex $v \in C_{k}$ is free if $v$ is incident to no chord of $C_{k}$ and if $v \notin C\left(B_{1}\right)$ (see Fig. 2 top left). The shelling procedure goes as follows ( $n$ is the number of vertices in $G \backslash C\left(B_{1}\right)$ ): for $k$ from $n$ to 1 , choose a free vertex $v$ on $C_{k}$, assign $v_{k} \leftarrow v$, and then delete $v$ together with all its incident edges. The existence of a free vertex at each step follows from the same argument as in the planar case [3]. Indeed, if there is no chord incident to $C_{k}$, then any vertex $v \in C_{k} \backslash C\left(B_{1}\right)$ is free, while if there is a chord $e$ for $C_{k}$, then the set of chords incident to $C_{k}$ forms a system of archs (relative to $C_{k}$ ). If we look at a chord $e=\{u, v\}$ that is "innermost" (i.e., seen as an arch, no other arch is nested inside), then the path between $u$ and $v$ on $C_{k}$ contains at least one vertex, which has to be free (see Fig. 2 top right).

Underlying Forest and Dual Forest. Given a cylindric triangulation $G$ (with boundary faces $B_{1}$ and $B_{2}$ ) endowed with a canonical ordering $\pi$, define the underlying forest $F$ for $\pi$ as the oriented subgraph of $G$ where each vertex $v \in C\left(B_{2}\right)$ has outdegree 0 , and where each $v \notin C\left(B_{2}\right)$ has exactly one outgoing edge, which is connected to the adjacent vertex of $v$ of largest label in $\pi$. The forest $F$ can be computed on the fly during the shelling procedure: when treating a free vertex $v_{k}$, for each neighbour $v$ of $v_{k}$ such that $v \notin C_{k}$, add the edge $\left\{v, v_{k}\right\}$ to $F$, and orient it from $v$ to $v_{k}$. Since the edges are oriented in increasing labels, $F$ is an oriented forest; it spans all vertices of $G \backslash C\left(B_{2}\right)$ and has its roots on $C\left(B_{2}\right)$. The augmented map $\widehat{G}$ is obtained from $G$ by adding a vertex $w_{1}$ inside $B_{1}$, a vertex $w_{2}$ inside $B_{2}$, and connecting all vertices around $B_{1}$ to $w_{1}$ and all vertices around $B_{2}$ to $w_{2}$ (thus triangulating the interiors of $B_{1}$ and $B_{2}$, see Fig. 2 bottom middle). Define $\widehat{F}$ as $F$ plus all edges incident to $w_{1}$ and all edges incident to $w_{2}$. Define the dual forest $F^{*}$ for $\pi$ as the graph formed by the vertices of $\widehat{G}^{*}$ (the dual of $\widehat{G}$ ) and by the edges of $\widehat{G}^{*}$ that are dual to edges not in $\widehat{F}$. Since $\widehat{F}$ is a spanning connected subgraph of $\widehat{G}, F^{*}$ is a spanning forest of $\widehat{G}^{*}$. Precisely, each of the trees (connected components) of $F^{*}$ is rooted at a vertex "in front of" each edge of $B_{1}$, and the edges of the tree can be oriented toward this root-vertex (see Fig. 2 bottom right).

Drawing Algorithm. Given a cylindric triangulation $G$ such that $C\left(B_{1}\right)$ has no chord, we first compute a canonical ordering of $G$, and then draw $G$ in an incremental way. We start with a cylinder of width $2\left|C\left(B_{1}\right)\right|$ and height 0 (i.e., a circle of length $2\left|C\left(B_{1}\right)\right|$ ) and draw the vertices of $C\left(B_{1}\right)$ equally spaced on the circle (space 2 between two consecutive vertices). Then the strategy for each $k \geq 1$ is to compute the drawing of $G_{k}$ out of the drawing of $G_{k-1}$ by first stretching the cylinder (increasing the width by 2) and then placing the vertex $v_{k}$ and its incident edges (in $G_{k}$ ) in a planar way. Define the $x$-span of an edge $e$ in the cylindric drawing as the number of columns 

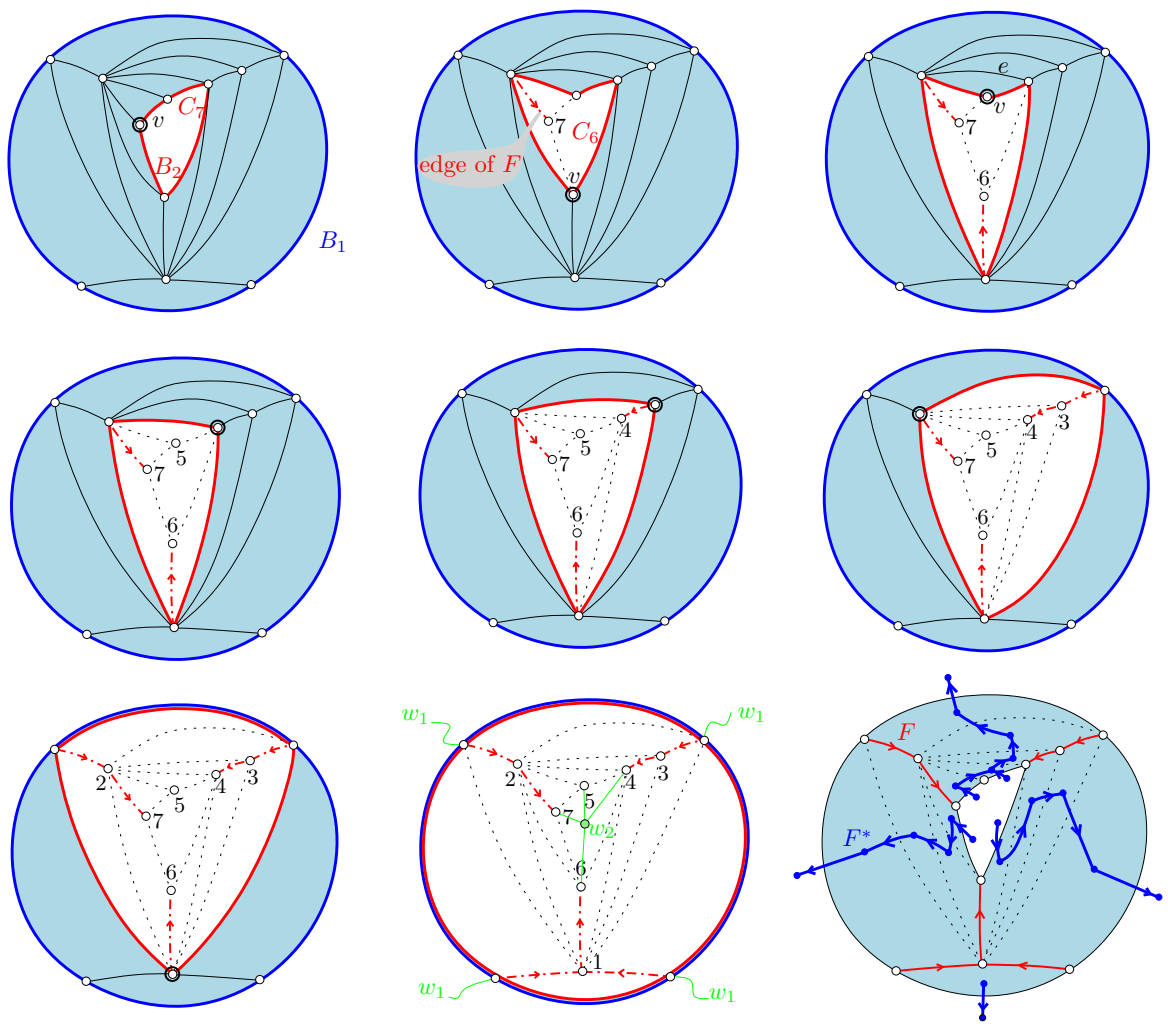

Fig. 2. Shelling procedure to compute a canonical ordering of a given cylindric triangulation. The underlying forest is computed on the fly; the last drawing shows the underlying forest superimposed with the dual forest. The graph is the one of Fig. 1

$[i, i+1] \times[0,+\infty]$ that meet the interior of $e$ (we have no need for a more complicated definition since, in our drawings, a column will never meet an edge more than once).

Consider the dual forest $F^{*}$ for the canonical ordering restricted to $G_{k-1}$. Note that the set of vertices of $C_{k-1}$ that are neighbours of $v_{k}$ form a path on $C_{k-1}$. Traversing this path $\gamma$ with the boundary-face of $G_{k-1}$ (the one different from $B_{1}$ ) to the left, let $e_{\ell}$ be the first edge of $\gamma$ and $e_{r}$ be the last edge of $\gamma$. Let also $a_{k}$ be the starting vertex and let $b_{k}$ be the ending vertex of $\gamma$. Let $P_{\ell}$ (resp. $P_{r}$ ) be the path in $F^{*}$ from $e_{\ell}^{*}$ (resp. $e_{r}^{*}$ ) to the root in its connected component (which is a vertex "in front of" an edge of $B_{1}$ )). We stretch the cylinder by inserting a vertical strip of length 1 along $P_{\ell}$ and another along $P_{r}$ (see Fig. 3). This comes down to increasing by 1 the $x$-span of each edge of $G_{k}$ dual to an edge in $P_{\ell}$, and then increasing by 1 the $x$-span of each edge dual to an edge in $P_{r}$ (note that $P_{\ell}$ and $P_{r}$ are not necessarily disjoint, in which case the $x$-span of an edge dual to an edge in $P_{\ell} \cap P_{r}$ is increased by 2). After these stretching operations, whose effect is to make the slopes of $e_{\ell}$ and $e_{r}$ strictly smaller than 1 in absolute value, we insert (as in the planar case) the vertex $v_{k}$ at the intersection of the ray of slope 1 starting from $a_{k}$ and the ray of slope -1 starting from $b_{k}$, and we connect $v_{k}$ to all vertices of 

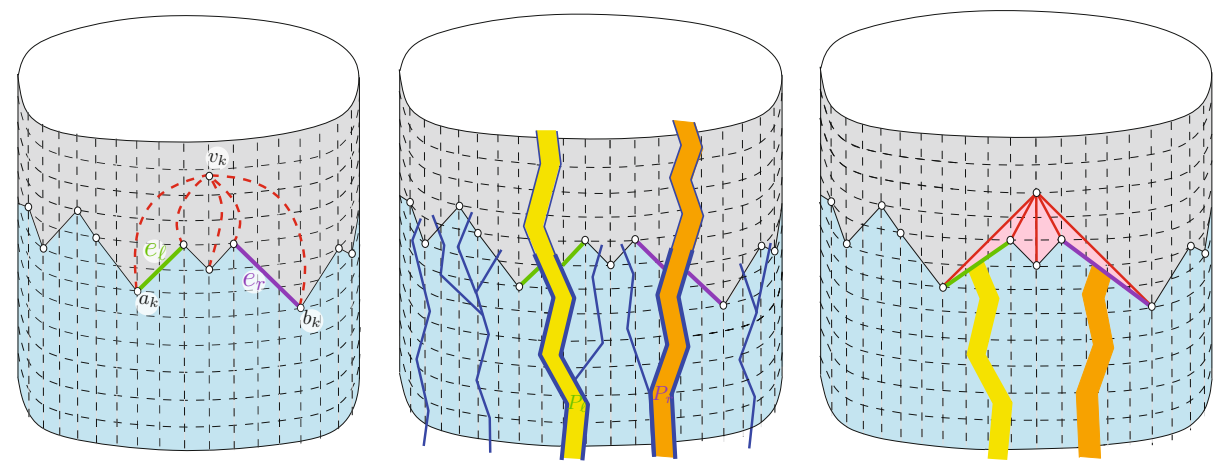

Fig. 3. One step of the incremental drawing algorithm. Two vertical strips of width 1 (each one along a path in the dual forest) are inserted in order to make the slopes of $e_{\ell}$ and $e_{r}$ smaller than 1 in absolute value. Then the new vertex and its edges connected to the upper boundary can be drawn in a planar way.

$\gamma$ by segment 1 . These two rays actually intersects at a grid point since the Manhattan distance between any two vertices on $C_{k-1}$ is even. Fig. 4 4 shows the execution of the algorithm on the example of Fig. 1.

Proposition 1. For each cylindric triangulation $G$ with no chord incident to $C\left(B_{1}\right)$, one can compute in linear time a crossing-free straight-line drawing of $G$ on an $x$ periodic regular grid $\mathbb{Z} / w \mathbb{Z} \times[0 . . h]$ where - with $n$ the number of vertices of $G$ and $d$ the (graph-)distance between the two boundaries $-w=2 n$ and $h \leq n(2 d+1)$, such that: every $v \in C\left(B_{1}\right)$ has $y(v)=0$ (so every edge in $C\left(B_{1}\right)$ has slope 0 ), and every edge belonging to $C\left(B_{2}\right) \backslash C\left(B_{1}\right)$ has slope \pm 1 .

Proof. The fact that the drawing remains crossing-free relies on the slope-property for the upper boundary and on the following inductive property, which is easily shown to be maintained at each step $k$ from 1 to $n$ :

Pl: for each edge $e$ on $C_{k}$ (the upper boundary of $G_{k}$ ), let $P_{e}$ be the path in $F^{*}$ from $e^{*}$ to the root, let $E_{e}$ be the set of edges dual to edges in $P_{e}$, and let $\delta_{e}$ be any nonnegative integer. Then the drawing remains planar when successively increasing by $\delta_{e}$ the $x$ span of all edges of $E_{e}$, for all $e \in C_{k}$.

We now prove the bounds on the grid-size. If $\left|C\left(B_{1}\right)\right|=t$ then the initial cylinder is $2 t \times 0$; and at each vertex insertion, the grid-width grows by 2 . Hence $w=2 n$. In addition, due to the slope conditions (slopes of boundary-edges are at most 1 in absolute value), the $y$-span (vertical span) of every edge $e$ is not larger than the current width at the time when $e$ is inserted in the drawing. Hence, if we denote by $v$ the vertex of $C\left(B_{2}\right)$

\footnotetext{
${ }^{1}$ In the FPP algorithm for planar triangulations, the step to make the (absolute value of) slopes of $e_{\ell}$ and $e_{r}$ smaller than 1 is formulated as a shift of certain subgraphs described in terms of the underlying forest $F$. The extension of this formulation to the cylinder would be quite cumbersome. We find the alternative formulation with strip insertions more convenient for the cylinder (it also gives rise to a very easy linear-time implementation).
} 


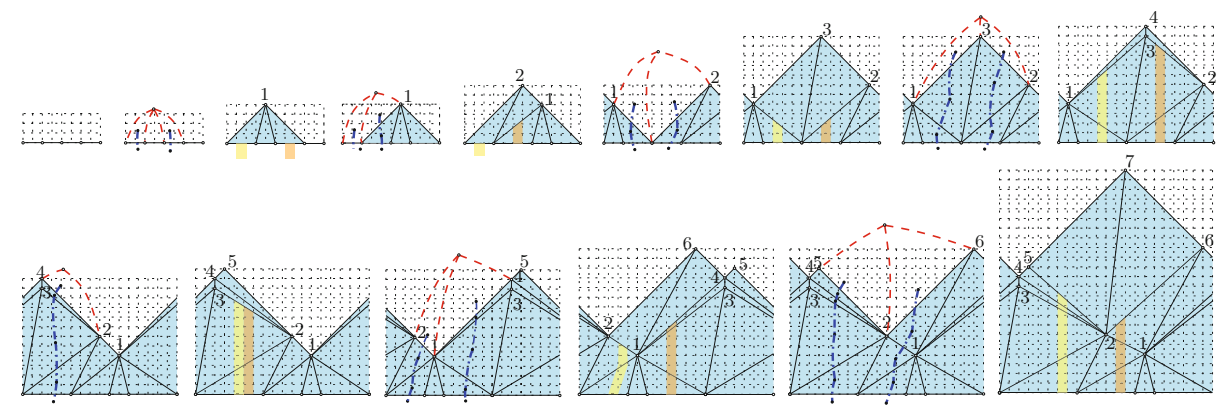

Fig. 4. Algorithm (Prop. 1) to compute an $x$-periodic drawing of a cylindric triangulation (no chordal edges incident to $B_{1}$ ). The vertices are treated in increasing label (the canonical ordering is the one computed in Fig. 2].

that is closest (at distance $d$ ) to $C\left(B_{1}\right)$, then the ordinate of $v$ is at most $d \cdot(2 n)$. And due to the slope conditions, the vertical span of $C\left(B_{2}\right)$ is at most $w / 2 \leq n$. Hence the grid-height is at most $n(2 d+1)$. The linear-time complexity is shown next.

Linear-Time Implementation. An important remark is that, instead of computing the $x$-coordinates and $y$-coordinates of vertices in the drawing, one can compute the $y$ coordinates of vertices and the $x$-span of edges (as well as the knowledge of which extremity is the left-end vertex and which extremity is the right-end vertex). In a first pass (for $k$ from 1 to $n$ ) one computes the $y$-coordinate of vertices and the $x$-span $r_{e}$ of each edge $e \in G$ at the time $t=k$ when it appears on $G_{k}$ (as well one gets to know which extremity of $e$ is the left-end vertex). Afterwards if $e \notin F$, the $x$-span of $e$ might further increase due to insertion of new vertices. Precisely, let $v_{j}$ be a vertex inserted afterwards (i.e., $j>t$ ), and (with the notations $a_{j}, b_{j}$ of the drawing algorithm description) let $\epsilon_{\ell}=\left\{v_{j}, a_{j}\right\}$ and $\epsilon_{r}=\left\{v_{j}, b_{j}\right\}$. Note that $e$ is stretched due to the insertion of the strip along $P_{\ell}$ iff $\epsilon_{\ell}^{*}$ is in the subtree $T_{e}$ of $F^{*}$ formed by the edges descending from $e^{*}$. Similarly $e$ is stretched due to the insertion of the strip along $P_{r}$ iff $\epsilon_{r}^{*}$ is in $T_{e}$. To state it more clearly, each edge in $T_{e}$ is responsible for an increase (by 1$)$ of the $x$-span of $e$. Hence the total $x$-span of each edge $e \in G$ is given by $r_{e}+s_{e}$, where $s_{e}=0$ if $e \in F$, and, if $e \notin F, s_{e}$ is the number of edges in $T_{e}$. Since all quantities $s_{e}$ can easily be computed in linear time, this gives a linear-time implementation.

Allowing for Chordal Edges at $\mathbf{C}\left(\mathbf{B}_{1}\right)$. We finally explain how to draw a cylindric triangulation when allowing for chords incident to $C_{0}=C\left(B_{1}\right)$; it is good to view $B_{2}$ as the top boundary-face and $B_{1}$ as the bottom-boundary face (and imagine a standing cylinder). For each chordal edge $e$ for the cycle $C_{0}$, the component under $e$ is the faceconnected part of $G$ that lies below $e$; such a component is a quasi-triangulation (polygonal outer face, triangular inner faces) rooted at the edge $e$. A chordal edge $e$ of $C_{0}$ is maximal if the component $Q_{e}$ under $e$ is not strictly included in the component under another chordal edge for $C_{0}$. The size of such an edge $e$ is defined as $|e|=2\left|V\left(Q_{e}\right)\right|-4$. (the size $|e|$ is actually the width of the drawing of $Q_{e}$ using the FPP algorithm). If we delete the component under each maximal chordal edge (i.e., delete everything 

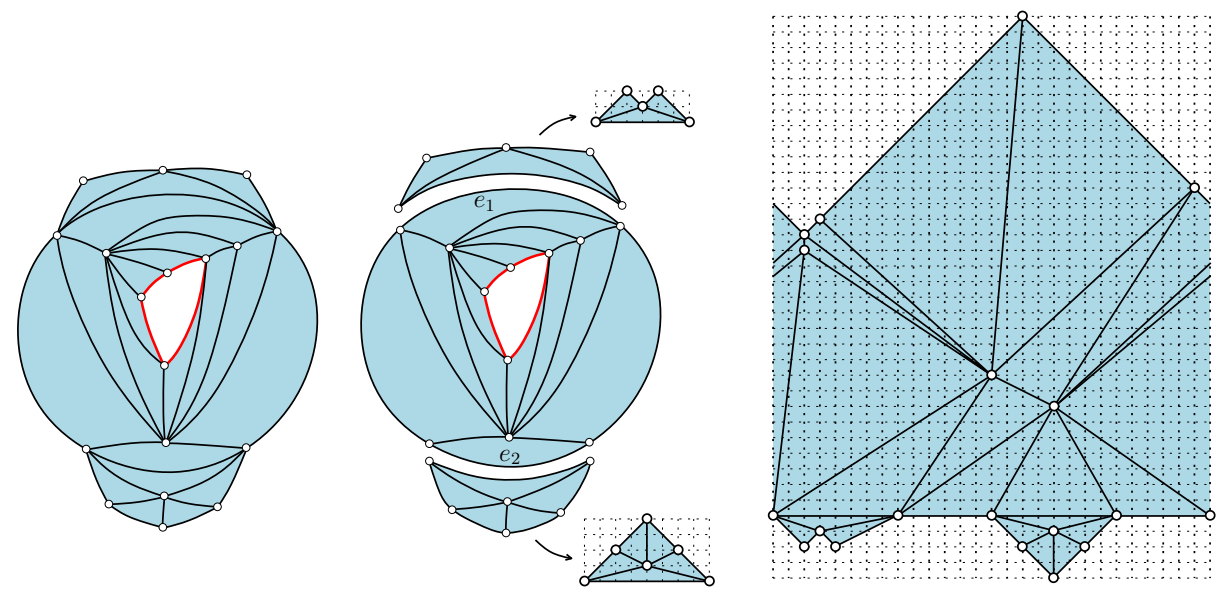

Fig. 5. Drawing a cylindric triangulation with chords at $B_{1}$. To make enough space to place the component under $e_{2}$, one takes 8 (instead of 2) as the initial $x$-span of $e_{2}$.

from the component except for the chordal edge itself) we get a new bottom cycle $C_{0}^{\prime}$ that is chordless, so we can draw the reduced cylindric triangulation $G^{\prime}$ using the algorithm of Proposition 1 As we have seen in Section 3 (implementation paragraph), for each edge $e$ of $C_{0}^{\prime}$, the initial $x$-stretch is $r_{e}=2$ and then the further increase $s_{e}$ of the $x$-stretch equals the number of edges descending from $e^{*}$ in the dual forest $F^{*}$. Note that we have actually some freedom to choose the initial $x$-stretch $r_{e}$ of each edge $e \in C_{0}^{\prime}$ (just it has to be a positive even number since at each step of the incremental algorithm the vertices of the current upper boundary have to be at even Manhattan distance). If $e \in C_{0}^{\prime}$ is on $C_{0}$ we take $r_{e}=2$. If $e \in C_{0}^{\prime}$ is not on $C_{0}$ (i.e., $e$ was a maximal chordal edge for $C_{0}$ ), we take for $r_{e}$ the minimal even positive number such that $r_{e}+s_{e} \geq|e|$, i.e., $r_{e}=2 \cdot \max \left(1,\left\lceil\left(|e|-s_{e}\right) / 2\right\rceil\right)$. Hence, at the end of the execution of the drawing of $G^{\prime}$, the length $\ell_{e}=r_{e}+s_{e}$ of each maximal chord $e$ satisfies $\ell_{e} \geq|e|$. Then for each maximal chord $e$ of $C_{0}$, we draw the component $Q_{e}$ under $e$ using the FPP algorithm. This drawing has width $|e|$, with $e$ as horizontal bottom edge of length $|e|$ and with the other outer edges of slopes \pm 1 . We shift the left-extremity of $e$ so that the drawing of $Q_{e}$ gets width $\ell(e)$, then we rotate the drawing of $Q_{e}$ by 180 degrees and plug it into the drawing of $G^{\prime}$ (see Fig. 5). The overall drawing of $G$ is clearly planar. We obtain:

Theorem 1. For each cylindric triangulation $G$, one can compute in linear time a crossing-free straight-line drawing of $G$ on an $x$-periodic regular grid $\mathbb{Z} / w \mathbb{Z} \times[0, h]$, where - with $n$ the number of vertices and $d$ the (graph-) distance between the two boundaries- $w \leq 2 n$ and $h \leq n(2 d+1)$. The drawing is $x$-monotone (the intersection with any vertical line is an interval) and the slopes of boundary-edges are at most 1 in absolute value. 


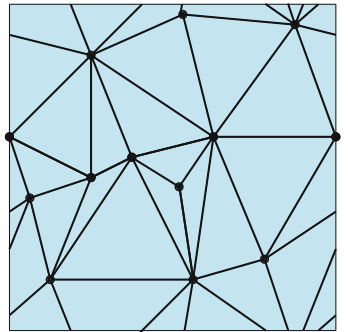

1)

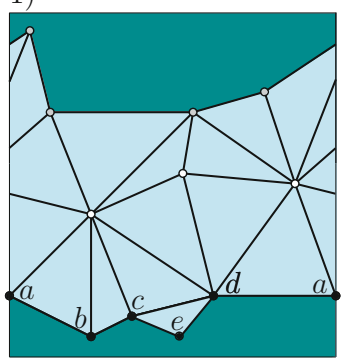

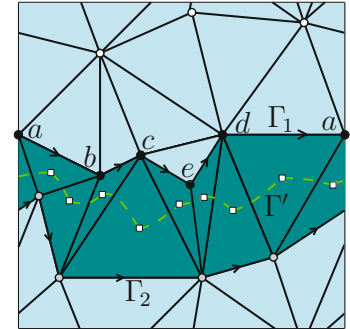

2)

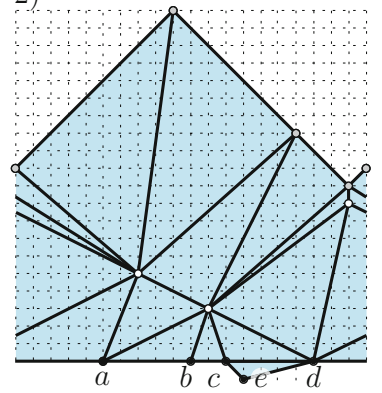

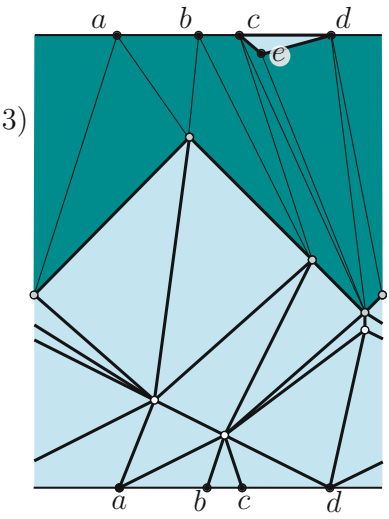

Fig. 6. The main steps for drawing of a toroidal triangulation: 1) remove the edges inside a tambourine, (2) draw the obtained cylindric triangulation, 3) insert the edges of the tambourine back into the drawing

\section{Periodic Drawings of Toroidal Triangulations}

Let $G$ be a toroidal triangulation. A non-contractible cycle of $G$ is a cycle of edges of $G$ that does not delimit a topological disk. Note that a pair $\Gamma_{1}, \Gamma_{2}$ of oriented noncontractible cycles that are parallel (i.e., are homotopic and are oriented in a parallel way) delimits a cylindric triangulation $T$, which is formed by the faces to the right of $\Gamma_{1}$ and to the left of $\Gamma_{2}$. The pair $\Gamma_{1}, \Gamma_{2}$ is called a tambourine if the edges dual to those of $T \backslash\left\{\Gamma_{1}, \Gamma_{2}\right\}$ form a non-contractible cycle $\Gamma^{\prime}$ that is homotopic to $\Gamma_{1}$ and $\Gamma_{2}$ (see darkblue faces and dashed edges in Fig. 6 top-middle). The edges of $T \backslash\left\{\Gamma_{1}, \Gamma_{2}\right\}$ are said to be inside the tambourine. It can be shown (see [2], the master's thesis of the third author Arnaud Labourel, and the next paragraph) that for each non-contractible cycle $\Gamma$ of $G$, there exists a tambourine whose two cycles are parallel to $\Gamma$. Deleting the edges that are strictly inside the tambourine, one obtains a cylindric triangulation $G^{\prime}$ with $\Gamma_{1}$ and $\Gamma_{2}$ as the contours of the boundary-faces. Note also that the distance $d$ between $\Gamma_{1}$ and $\Gamma_{2}$ is smaller than the length of a shortest non-contractible cycle not parallel to $\Gamma$. We now apply the algorithm of Theorem 1 to $G^{\prime}$. If we augment the height $h$ of the drawing to $h^{\prime}=h+w+1$, and then wrap the $x$-periodic grid $\mathbb{Z} / w \mathbb{Z} \times[0 . . h]$ into a periodic $\operatorname{grid} \mathbb{Z} / w \mathbb{Z} \times \mathbb{Z} / h^{\prime} \mathbb{Z}$, and finally insert the edges inside the tambourine as segment 2 ,

\footnotetext{
${ }^{2}$ We insert the edges in the tambourine $T$ in the unique way such that, looking from bottom to top, at least one edge in $T$ goes strictly to the right, and all edges going strictly to the right have $x$-span at most $w$; in this way it is easy to check that the $x$-span of all edges in $T$ is at most $w$.
} 
then the slope properties (edges on $\Gamma_{1}$ and $\Gamma_{2}$ have slope at most 1 in absolute value while edges inside the tambourine have slopes greater than 1 in absolute value) ensure that the resulting drawing is crossing-free (see Fig. 6). Observe that we can choose $\Gamma$ so that the graph-distance between the two boundaries $\Gamma_{1}$ and $\Gamma_{2}$ (in $G^{\prime}$ ) is smaller than the length $\gamma$ of a shortest non-contractible cycle in $G$; and this choice for $\Gamma$ can be done without computing a shortest non-contractible cycle. Indeed, let $\left\{\Gamma_{a}, \Gamma_{b}\right\}$ be a basis of non-contractible cycles (computable in linear time, using for instance a cut-graph). Denoting by $\Gamma_{\min }$ a shortest non-contractible cycle of $G$, for sure at least one of $\Gamma_{a}$ or $\Gamma_{b}$ is not parallel to $\Gamma_{\min }$. Hence, for $\Gamma_{a}$ or for $\Gamma_{b}$, the distance between the boundary-cycles (after deleting edges of the parallel tambourine) is smaller than $\left|\Gamma_{\min }\right|$. In other words if we choose the one cycle (among $\left\{\Gamma_{a}, \Gamma_{b}\right\}$ ) that yields the smaller distance between the two boundaries of $G^{\prime}$, then this distance will be smaller than $\gamma$. We obtain:

Theorem 2. For each toroidal triangulation $G$, one can compute in linear time a crossing-free straight-line drawing of $G$ on a periodic regular grid $\mathbb{Z} / w \mathbb{Z} \times \mathbb{Z} / h \mathbb{Z}$, where (with $n$ the number of vertices and $\gamma$ the length of a shortest non-contractible cycle) $w \leq 2 n$ and $h \leq 1+n(2 \gamma+1)$. Since $\gamma \leq \sqrt{2 n}$ (as shown in [1]), the grid area is $O\left(n^{5 / 2}\right)$.

Existence of a Tambourine. For the sake of completeness we include a proof of existence of a tambourine, which slightly extends the proof given in the master's thesis of Arnaud Labourel. A toroidal map is called essentially 3-connected if its periodic representation in the plane is 3-connected. Let $G$ be such a map and let $\Gamma$ be a noncontractible cycle of $G$. We are going to show that $G$ has a tambourine parallel to $\Gamma$. Let $G^{\prime}$ be the cylindric map obtained after cutting $G$ along $\Gamma$; we take the annular representation of $G^{\prime}$, calling $\Gamma_{1}$ (resp. $\Gamma_{2}$ ) the copy of $\Gamma$ that is the outer (resp. inner) boundary. Let $\Gamma^{\prime}$ be the smallest (in terms of the enclosed area) cycle that strictly encloses $\Gamma_{2}$ (i.e., encloses $\Gamma_{2}$ and is vertex-disjoint from $\Gamma_{2}$ ). Let $\Gamma^{\prime \prime}$ be the largest (in terms of the enclosed area) cycle that is strictly enclosed in $\Gamma^{\prime}$ (i.e., is enclosed by $\Gamma^{\prime}$ and is vertex-disjoint from $\Gamma^{\prime}$ ). Note that by minimality $\Gamma^{\prime}$ has no chord inside, and by maximality $\Gamma^{\prime \prime}$ has no chord outside. Hence, if we can show that there is no vertex in the area $A$ (strictly) between $\Gamma^{\prime}$ and $\Gamma^{\prime \prime}$, then we can conclude that, in $G, \Gamma^{\prime}$ and $\Gamma^{\prime \prime}$ form a tambourine parallel to $\Gamma$. Assume there is a vertex $v$ in $A$. Call vertex of attachment for $\Gamma^{\prime}$ a vertex $w \in \Gamma^{\prime}$ such that there is a path from $v$ to $w$ visiting only vertices of $A$ before reaching $w$. Again by minimality of $\Gamma^{\prime}$ it is easy to see that there is a unique vertex of attachment $v^{\prime}$ for $\Gamma^{\prime}$. Similarly (by maximality of $\Gamma^{\prime \prime}$ ) there is a unique vertex of attachment $v^{\prime \prime}$ for $\Gamma^{\prime \prime}$. Then (again by minimality of $\Gamma^{\prime}$ and maximality of $\Gamma^{\prime \prime}$ ) there is a closed curve $\gamma$ that meets $G^{\prime}$ only at the vertices $v^{\prime}, v^{\prime \prime}$, and such that the interior of $\gamma$ contains $v$ but does not contain any of the two boundary-faces. Such a curve $\gamma$ yields a 2 -separator in the periodic representation of $G$, a contradiction.

\section{Allowing for Non-contractible 1- and 2-Cycles}

For a cylindric map, a non-contractible cycle is a cycle that separates the two boundaryfaces (i.e., there is a boundary-face on one side and a boundary-face on the other side 


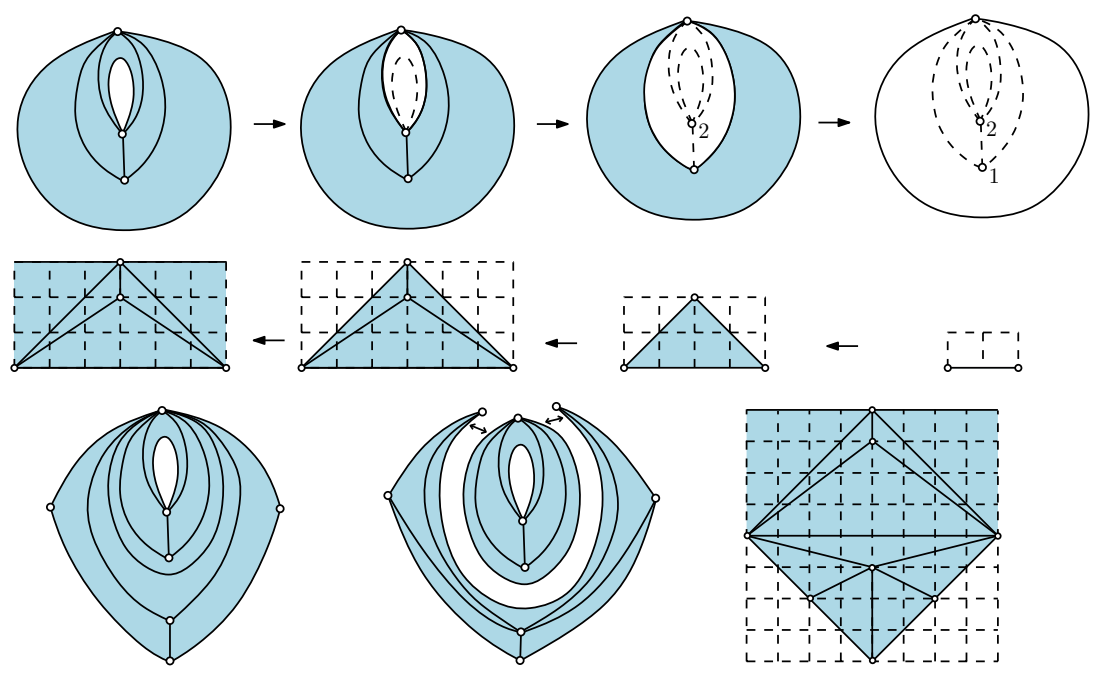

Fig. 7. Top line: canonical ordering of an essentially-simple cylindric triangulation (with some non-contractible 1- and 2-cycles). Middle line: iterative drawing algorithm. Bottom line: dealing with chordal edges.

of the cycle). Other cycles are called contractible. An essentially-simple cylindric triangulation is a cylindric map whose non-boundary faces are triangles, whose contractible cycles have length at least 3 , and such that each vertex has at most one incident noncontractible loop. An essentially-simple toroidal triangulation is a toroidal map with triangular faces, with all contractible cycles of length at least 3 , and such that any two (non-contractible) loops incident to the same vertex can not be homotopic. These are the necessary and sufficient conditions for the triangulation (whether on the cylinder or on the torus) to have no loops nor multiple edges in the periodic representation; hence these are the conditions under which one can aim at a periodic crossing-free straightline drawing. For essentially-simple cylindric triangulations without loops (nor chords incident to $C\left(B_{1}\right)$ ), exactly the same shelling procedure and iterative drawing algorithm can be taken as for simple cylindric triangulations. In case there are loops we have to explain how to deal with them (see Fig.7 for an example). For the shelling procedure, if the current upper boundary $C_{k}$ is a loop - call $v$ the incident vertex — then one deletes the loop and immediately takes $v$ as the next free vertex (the fact that $v$ is free is due to the fact that there is no other loop at $v$ ). In the drawing procedure (how to insert $v$ and its incident loop into the drawing), one first adds $v$ without its loop (by a classical one-step iteration of the drawing algorithm, involving two strip insertions), and then one draws the loop at $v$ as an horizontal segment spreading over the whole width of the current periodic drawing (the loop is added without inserting any vertical strip). Finally one can deal with chords incident to $C\left(B_{1}\right)$ in the same way as for simple cylindric triangulations. About essentially-simple toroidal triangulations, the procedure is also the same as for simple toroidal triangulations since the above proof of existence of a 
tambourine holds in that case. And the grid bounds (for the cylindre and for the torus) are the same as for simple triangulations.

Acknowledgments. The authors thank D. Gonçalves and B. Lévêque, and (independently) B. Mohar for interesting discussions about toroidal Schnyder woods, and N. Bonichon for explanations on the computation of a tambourine in a toroidal triangulation.

\section{References}

1. Albertson, M.O., Hutchinson, J.P.: On the independence ratio of a graph. J. Graph. Theory 2, 1-8 (1978)

2. Bonichon, N., Gavoille, C., Labourel, A.: Edge partition of toroidal graphs into forests in linear time. In: ICGT, vol. 22, pp. 421-425 (2005)

3. Brehm, E.: 3-orientations and Schnyder 3-trees decompositions, Master's thesis, FUB (2000)

4. Castelli-Aleardi, L., Fusy, E., Lewiner, T.: Schnyder woods for higher genus triangulated surfaces, with applications to encoding. Discr. \& Comp. Geom. 42(3), 489-516 (2009)

5. Chambers, E., Eppstein, D., Goodrich, M., Loffler, M.: Drawing graphs in the plane with a prescribed outer face and polynomial area. JGAA 16(2), 243-259 (2012)

6. de Fraysseix, H., Pach, J., Pollack, R.: How to draw a planar graph on a grid. Combinatorica 10(1), 41-51 (1990)

7. Duncan, C., Goodrich, M., Kobourov, S.: Planar drawings of higher-genus graphs. Journal of Graph Algorithms and Applications 15, 13-32 (2011)

8. Gonçalves, D., Lévêque, B.: Toroidal maps: Schnyder woods, orthogonal surfaces and straight-line representations arXiv:1202.0911 (2012)

9. Gortler, S.J., Gotsman, C., Thurston, D.: Discrete one-forms on meshes and applications to 3D mesh parameterization. Computer Aided Geometric Design 23(2), 83-112 (2006)

10. Kant, G.: Drawing planar graphs using the canonical ordering. Algorithmica 16(1), 4-32 (1996)

11. Kocay, W., Neilson, D., Szypowski, R.: Drawing graphs on the torus. Ars Combinatoria 59, 259-277 (2001)

12. Mohar, B.: Straight-line representations of maps on the torus and other flat surfaces. Discrete Mathematics 15, 173-181 (1996)

13. Mohar, B., Rosenstiehl, P.: Tessellation and visibility representations of maps on the torus. Discrete \& Comput. Geom. 19, 249-263 (1998)

14. Schnyder, W.: Embedding planar graphs on the grid. In: SODA, pp. 138-148 (1990)

15. Zitnik, A.: Drawing graphs on surfaces. SIAM J. Disc. Math. 7(4), 593-597 (1994) 